\title{
THE ORBITAL PARAMETERS AND THE IUE SPECTRUM OF THE SINGLE-LINED SPECTROSCOPIC BINARY HD 698
}

\author{
J. SAHADE ${ }^{1,2, *}$, R. BARBA ${ }^{1, \#}$ and Y. KONDO ${ }^{3}$ \\ 1. C.C. 677, Observatorio Astronómico \\ 1900 La Plata, Argentina \\ 2. Instituto Argentino de Radioastronomía \\ C.C. 5, 1894 Villa Elisa, Argentina \\ 3. Code 684, NASA Goddard Space Flight Center \\ Greenbelt, MD 20771, U.S.A.
}

The study that we have undertaken of HD 698 essentially provides:

1) a new determination of the orbital elements of the system on the basis of the available ground-based observations of Struve and Rudkjobing (1948), Sahade (1967) and Hutchings and Bernard (1978);

2) an analysis of four IUE images of the star, two secured in the high resolution mode and two secured with low resolution, that cover the wavelength range 1165-3230 $\AA$.

The ultraviolet observations:

a) suggest that the primary component of the system is a B5 III-II star;

b) show that, even in the ultraviolet, we are dealing with a single-lined binary;

c) confirm the presence of an extended circumbinary envelope in the system;

d) suggest the presence of non-thermal sources in the system;

e) suggest that the extended envelope must start expanding at a certain distance from the star and begins to decelerate somewhere between the layer where He II 1640 is formed and the layer where the resonance doublet of Si IV originates, reaching, at the boundary, velocity values similar to those of the interstellar medium.

The paper in full will be published elsewhere.

\section{References}

Hutchings, J. B. and Bernard, J. E. 1978, P.A.S.P. 90, 179.

Sahade, J., 1967 in Modern Astrophysics, ed. M. Hack (Gauthier-Villars), p. 219.

Struve, O. and Rudkjobing, M. 1948, Ap. J. 108, 537.

* Member of the Carrera del Investigador Cientifico, CONICET, Argentina.

\# On a Fellowship from CONICET, Argentina. 